Aus der pädiatrischen Klinik (Vorstand: Prof. Es cherich) und dem pathologisch-anatomischen Institut (Vorstand: Prof. We ich selba m) in Wien.

\title{
Über einen Fall von Prolaps der Harnblase bei Pertussis.
}

Von Dr. Waichi Hirokawa aus Tokyo.

(Mit I Abbildung.)

Am 26. April I9Io wurde das 8 Monate alte Mädchen M. H. mit dem Befunde eines Prolapses der Harnblase auf die pädiatrische Klinik aufgenommen.

Aus der Anamnese ergab sich, da $B$ das Kind normal geboren wurde und bis vor 3 Monaten gesund war. Damals bekam das Mädchen Husten vom Charakter des Hustens bei Pertussis, der seitdem in gleicher Intensität anhielt.

Am 23. März, also ungefähr einen Monat vor der Aufnahme in das Spital, bemerkte die Mutter, daß während eines Hustenanfalles beim Kinde in der Schamspalte eine rote Geschwulst von der Größe eines Kirschkernes hervortrat. Seitdem zeigte sich dieses Gebilde bei jedem Hustenanfall und trat nach Beendigung des Anfalls wieder zurück. $\mathrm{Ob}$ zu dieser Zeit Harnträufeln bestand oder nicht, konnte die Mutter nicht angeben. Bemerkt wurde jedoch, daß das Kind nicht of trocken gelegt werden konnte.

Am 25. April konstatierte die Mutter, daß die Geschwulst nach dem Hustenanfalle nicht mehr zurücktrat; seither war das Kind immer naß.

Das Mädchen, das ein Brustkind war, hatte bei der Aufnahme ein Gewicht von 5roo g, war ziemlich mager und anämisch, zeigte noch keine Zähne und machte keinen Versuch sich aufzusetzen. Die Knochenränder an den Fontanellen waren federnd, die Rippen rosenkranzartig aufgetrieben und die Epiphysen verdickt. 
Das Mädchen zeigte krampfartige Hustenanfälle, Rasselgeräusche über den Lungen und Nasenflügelatmen.

$Z$ wischen den Beinen war ein ungefähr walnußgroßer, lebhaft geröteter Tumor sichtbar, an dessen unteren Hälfte beide Ureterenmündungen nachweisbar waren. Der Nabel und die Bauchwand zeigten keine Veränderungen. Die Rektalöffnung lag an der gewöhnlichen Stelle.

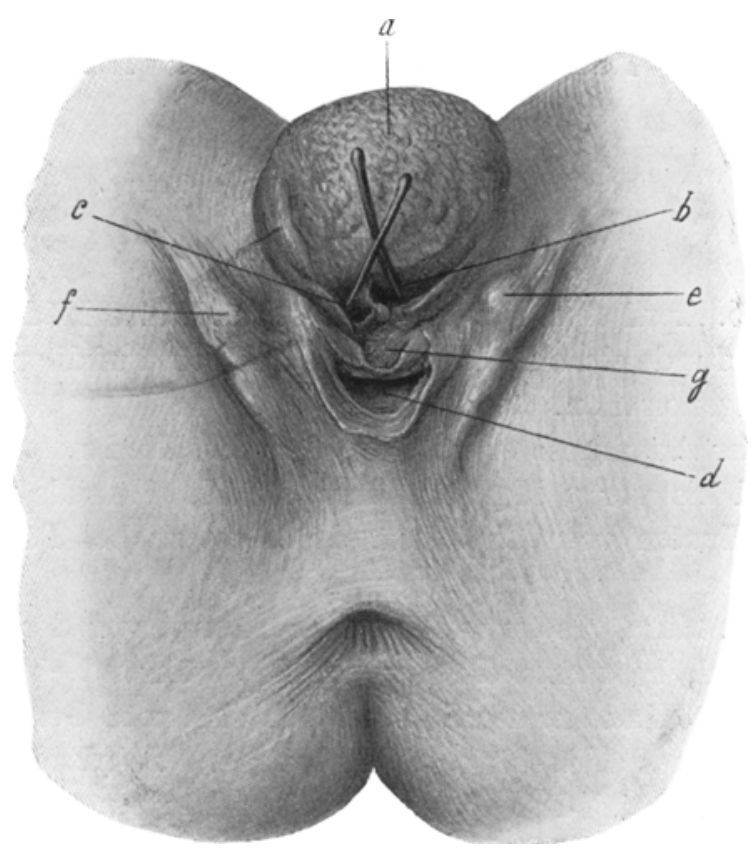

a) $=$ Prolaps $; b)=$ linke Uretermündung; $c$ ) $=$ rechte Uretermündung; $d=$ Vagina;

e) $=$ linke Hälfte der Clitoris; f) = rechte Hälfte der Clitoris; g) = Epispadie.

Eine Reposition des Tumors war unmöglich; auch der Versuch, den Tumor in Chloroformnarkose zu reponieren, blieb erfolglos.

Ein chirurgischer Eingriff konnte wegen des elenden Zustandes des Kindes nicht mehr vorgenommen werden.

Am 27. April starb das Mädchen. Bei der Sektion (Dr. A. $\mathrm{G} h \circ \mathrm{n}$ ) wurde folgender Befund erhoben:

$70 \mathrm{~cm}$ lange weibliche Kindesleiche, entsprechend entwickelt und mäßig gut genährt. 
Unter der Symphyse findet sich im Bereiche der obern Hälfte des äußern Genitales ein rundliches Gebilde von $2 \mathrm{~cm}$ Durchmesser und schmutzigrotem, samtartigem Aussehen, dessen untere Hälfte von graugelblichen, fibrinösen Exsudatmassen bedeckt ist.

In der Mitte seiner untern Hälfte zeigt dieses Gebilde eine schmale, leistenförmige Erhebung, die sich nach der Kuppe zu verliert, nach der Basis zu aber in einen $0,8 \mathrm{~cm}$ langen und $0,6 \mathrm{~cm}$ breiten, bandförmigen Schleimhautstreifen übergeht, der am oberen Rande des Vestibulum vaginae endet, dort wo unter gewöhnlichen Verhältnissen das Orificium urethrae externum $z u$ finden ist. Begrenzt wird dieser Schleimhautstreifen von zwei niedrigen Leisten, die das tumorartige Gebilde an seiner untern Hälfte seitlich umfassen und gleichfalls schmutzigrot sind.

Die Teile des Genitales, die als Labia minora pudendi angesprochen werden können, sind so zueinander gestellt, daß ihre obern Enden $3 \mathrm{~cm}$ breit klaffen und zwischen sich das tumorartige Gebilde in seiner untern Hälfte aufnehmen. Sie stellen grauweißlich glänzende, nach außen umgelegte, faltige Gebilde dar, von denen das rechte $I, 8 \mathrm{~cm}$, das linke $2 \mathrm{~cm}$ in der Länge mißt; ungefähr in der Mitte jhres äußeren Randes zeigen sie eine kleine Einkerbung und dieser entsprechend sieht man an der umgelegten vordern Fläche eine fast rundliche, flache, knotige und ziemlich derbe Erhebung, die fast $4 \mathrm{~mm}$ im Durchmesser hat; von diesen Knoten zieht eine schmale Schleimhautfalte fast horizontal gegen das tumorartige Gebilde hin, während sich die obere Hälfte der als kleine Labien angesprochenen Gebilde nach oben und außen

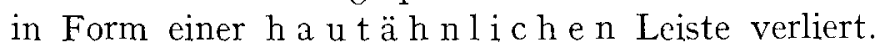

Die großen Schamlippen erscheinen als flache Wülste, die ohne scharfe Grenze in die Umgebung übergehen, nach innen zu aber von den nach außen umgelegten kleinen Labien zum Teil bedeckt sind.

Das Vestibulum vaginae ist sehr geräumig, der Hymen schmal und fast dreieckig geformt, seine Spitze nach der hintern Kommissur gerichtet. Die gleiche Form zeigt auch das Orificium vaginae. Die obere Wand der Vagina hängt leicht konvex nach vorne unten. - Die hintere Kommissur der Labien ist breit und flach, die Fossa navicularis fast verstrichen. Die hintern Enden der Labia minora klaffen $\mathrm{I}, 2 \mathrm{~cm}$ weit und umfassen nicht mehr den untern Teil des Vestibulums. 
Eine Öffnung, die als Orificium urethrae externum angesprochen werden konnte, ließ sich nicht nachweisen; ebenso fehlte an der gewöhnlichen Stelle die Clitoris.

Das Perinäum ist breit, die Analöffnung an normaler Stelle und durchgängig.

An der untern Hälfte des tumorartigen Gebildes finden sich in den Winkeln, die von der früher beschriebenen mittlern und den beiden seitlichen Schleimhautleisten gebildet werden, zwei kleine trichterförmige Vertiefungen, die in die beiden Ureteren führen. Diese ziehen in fast gerader Richtung zu den beiden Nieren und sind leicht erweitert; ihre Schleimhaut ist stark gerötet. Noch stärker gerötet erscheint die Schleimhaut der Nierenbecken und Nierenkelche, die gleichfalls erweitert sind und trüben Harn enthalten. Die Nieren sind entsprechend groß und zeigen Andeutung embryonaler Lappung; ihre Kapsel ist leicht abziehbar, ihre Oberfläche zeigt zahlreiche, leicht erhabene, graugelbe Eiterherde; zwischen diesen Herden ist das Gewebe rötlichgrau. Auf der Schnittfläche sieht man solche streifenförmige Herde in der Rinde und stellenweise auch in den Pyramiden. Die Basis der Pyramiden ist stark gerötet.

Der Uterus, der entsprechend groß erscheint, steht auffallend tief und ist anteflektiert, so daß seine hintere Fläche fast ganz nach oben und vorne gelagert und dadurch die Excavatio rectouterina wenig ausgebildet erscheint. Hingegen ist die vordere Excavatio tief und schmal. Die Harnblase, die sie sonst nach vorne zu begrenzt, fehlt hier; es findet sich unter dem Peritonealüberzug der vordern Wand der Excavatio nur Fettgewebe.

Die Adnexe sind ohne besondere Veränderungen.

Die Symphyse zeigt einen $2,5 \mathrm{~cm}$ breiten Spalt, der durch ein straffes, bindegewebiges, $0,5 \mathrm{~cm}$ breites Band überbrückt ist.

Die beiden Darmbeinschaufeln sind steil gestellt, ebenso das Kreuzbein, dessen hintere Fläche fast in einer Linie mit der Wirbelsäule steht.

Der Magendarmtraktus zeigt keine besonderen Veränderungen.

Die Milz ist $7,5 \mathrm{~cm}$ lang und $5,5 \mathrm{~cm}$ breit und zeigt auf der Schnittfläche zahlreiche kleine Follikel. 
Die Leber hat am hintern Rande des rechten Lappens eine tiefe Kerbe, ist sonst aber ohne besondere Veränderungen.

Die Thymus ist zweilappig und ziemlich substanzarm.

Beide Lungen sind frei, die Lingula des linken Oberlappens durch eine tiefe Furche markiert. In den hintern Partien beider Unterlappen und in den hintern obern des rechten Oberlappens finden sich größere atelektatische Herde, im linken Unterlappen außerdem bronchopneumonische Herde. Die Bronchien enthalten graues Sekret.

Das Herz ist entsprechend groß, sein Klappenapparat zart und schlußfähig, sein Foramen oval durchgängig.

Der lymphatische Apparat in der Mund- und Rachenhöhle ist kaum vergrößert.

Das Gehirn zeigt keine besonderen Veränderungen.

Die Diaphysen beider Oberschenkel sind dünn, ihre Epiphysen stark aufgetrieben; auf der Schnittfläche zeigt die chondrale Ossifikationszone typische rhachitische Veränderungen; den gleichen Befund weisen auch die rosenkranzartigen Verdickungen der Rippen an ihrer Knorpelgrenze auf.

Nachdem die Weichteile des Beckens abpräpariert und im Zusammenhange herausgenommen waren, gelang die Reposition des tumorartigen Gebildes ziemlich leicht: Es fand sich dann eine Offnung ungefähr für die Kuppe des kleinen Fingers durchgängig, die zunächst als das erweiterte Orificium urethrae externum imponierte; doch fehlte über dieser Offnung die Clitoris und die vordere Kommissur. Eine Fissur der Harnblase war jedoch nicht erkennbar.

Der Sagittalschnitt, der durch die in Formol gehärteten Beckenorgane geführt wurde, zeigte folgende Verhältnisse: Über dem Vestibulum vaginae springt das tumorartige Gebilde kugelig vor. Vom Vestibulum trennt es die seichte trichterförmige Einziehung, die in die Ureteren führt. Oben setzt sich das Gebilde scharf von der Bauchwand ab. Die Schnittfläche des Gebildes erscheint fast kompakt muskulös, in der äußern Schichte stark gerötet. In die Basis dieses Gebildes zieht ein feiner, kaum erkennbarer Spalt als Fortsetzung der hervorgezogenen, durch den anteflektierten Uterus sehr schmalen Excavatio vesico-uterina. Die vordere Fläche des Uterus erscheint demnach in ihrer 
ganzen Ausdehnung vom Peritoneum überzogen; eine Plica uterovesicalis und ein Spatium uterovesicale sind nicht erkennbar. Oben setzt sich das tumorartige Gebilde scharf von der Bauchwand $a b$ und zeigt an seiner Basis einige kleine zirkuläre Faltenbildungen.

Stufenschnitte, die durch das Labium minus der linken Seite geführt wurden und vor allem die beschriebene knotige Erhebung desselben trafen, zeigten unter einer Lage geschichteten Pflasterepithels zunächst eine dünne Schichte ziemlich zellreichen Bindegewebes, das nach der Tiefe zu reichliche Gefäße aufweist, woran sich ein fast ovales, mehr oder weniger deutlich umgrenztes Gebilde anschließt, das histologisch dem Bau der Corpora cavernosa clitoridis vollkommen entspricht: man sieht ein Maschenwerk von Bälkchen, die vorwiegend aus glatten Muskelzellen bestehen und sich in verschiedenen Richtungen verflechten, und darin eingeschlossen zahlreiche, meistens spaltförmige, leere Räume verschiedener Größe, die eine einfache Endothelauskleidung zeigen, auf der stellenweise rote Blutkörperchen gelagert sind. Auch einige größere Gefäße mit deutlicher muskulöser Wand findet man in diesem Gebilde und in der nächsten Umgebung desselben zahlreiche Durchschnitte von Nerven.

Es unterliegt demnach keinem $Z$ weifel, daß die knotenförmigen Erhebungen der Labia minora der geteilten Clitoris entsprechen.

Ein Schnitt, der durch die Mitte des tumorartigen Gebildes und des Genitales sagittal geführt wurde, zeigte, daß dieses tumorartige Gebilde in seinen basalen und zentralen Anteilen aus Muskelbündeln aufgebaut ist, zwischen denen Bindegewebe, stark dilatierte Gefäße und Rundzelleninfiltrate sichtbar sind. Der äußere Mantel des Gebildes besteht aus einer stark gequollenen bindegewebigen Schichte, die ganz durchsetzt ist von verschieden großen Blutungen und Rundzellen, sowie von stark erweiterten und prall gefüllten Gefäßen. Die äußersten Lagen zeigen stellenweise außerdem ein Balkenwerk von Fibrin mit zelligem Exsudat und zahlreichen großen Bakterienhaufen. Epithel ist nur am Ubergang des tumorartigen Gebildes in die Haut als geschichtetes Pflasterepithel erhalten.

Uberblicken wir den ausführlich beschriebenen Fall, so unterliegt es keinem $Z$ weifel, daß es sich einerseits um einen Prozeß 
handelt, der als Mißbildung aufzufassen ist, anderseits um einen Prozeß, der als ein erworbener Zustand angesehen werden muß. Der Mißbildung gehört das Spaltbecken an und die Veränderung am äußeren Genitale: Die vollständige $Z$ weiteilung der Clitoris und des Präputium clitoridis, die Anomalie der Urethra und der vorderen Kommissur und die abnorme Lagerung der kleinen Schamlippen.

Erworben ist zweifellos der Prolaps der Harnblase, der durch das oben beschriebene tumorartige Gebilde über dem Genitale dargestellt wird. Anamnestisch geht sicher hervor, daß der Prolaps erst im 7 . Lebensmonate von der Mutter bemerkt wurde und zwar im Anschlusse an eine Pertussiserkrankung. Anfangs klein, hat sich der Prolaps der Harnblase im Laufe des 8. Lebensmonates nach und nach zu der oben angegebenen Größe entwickelt. Eine Spaltbildung der Bauchwand und der Blase war nicht bemerkt worden, was ohne weiteres verständlich erscheint, da auch an der Leiche nach der Reposition des Prolapses nur eine runde Öffnung erkennbar war, die als erweitertes Orificium urethrae externum angesprochen werden konnte. Es handelte sich also um einen erworbenen Prolapsus vesicae urinariae bei vorhandenem Spaltbecken und bei einer Mißbildung des äußeren Genitales, die als E p is pa di e aufgefaßt werden muß. Nach der Einteilung von D urand wäre unser Fall als totale Epispadie (3. Grad) aufzufassen. Er entspricht übrigens allen Anforderungen für die weibliche Epispadie, wie sic K e r ma u ner im Handbuch von Ernst Schwalbe bei der Mißbildung des Rumpfes aufgestellt hat.

In der Literatur waren mir alle Originalarbeiten über ähnliche Fälle leider nicht zugänglich. Ich verweise daher auf die Arbeit von $\mathrm{R}$ a s c h, der die beschriebenen Fälle zusammengestellt hat. Ein genauer Vergleich meines Falles mit den in der Arbeit von $\mathrm{R}$ a s c h angeführten ist leider unmöglich, da nicht alle Details der beobachteten Veränderungen angegeben sind. So viel aber entnehme ich, da $B$ in dem von $L$ ow e beschriebenen Falle die Ausstülpung der Blase, die leicht reponiert werden konnte, eine angeborene war. Ob derProlapsus vesicae im Falle von $\mathrm{C}$ r o s s e angeboren oder erworben war, kann ich nicht beurteilen. Ebenso fehlt eine Angabe darüber im Falle von Oliver, bei dem so 
wie im Falle von $\mathrm{C}$ ros se die Reposition mühelos gelang. Auch der Fall von $W$ e in 1 e c h n e r, der ein 9 Monate altes Mädchen betraf, zeigte einen reponiblen Harnblasenprolaps, doch konnte ich aus der Arbeit nicht entnehmen, ob der Prolaps angeboren oder erworben war.

Im Falle von F r u a, der ein 6 Monate altes Mädchen betraf, erfolgte der Prolaps der Harnblase im Verlaufe einer Dysenterie; er konnte nicht mehr reponiert werden. Auch im Falle von $\mathrm{B}$ u n k e erfolgte der Blasenprolapsus bei einem II Monate alten, vorher ganz gesunden Mädchen nach einer erschwerten Stuhlentleerung bei hartnäckiger Obstipation. Der Fall von I m e r v a 1 endlich betraf ein 2 jähriges Mädchen, das einen Blasenprolaps nebst Mastdarmprolaps im Anschluß an Diarrhöe bei Masern acquirierte; auch hier gelang die Reposition.

Erwähnt sei noch, dæl $B$ auch in späteren Lebensjahren Harnblasenprolapse acquiriert werden können: T h o $\mathrm{m}$ s o $\mathrm{n}$ beschreibt einen solchen Fall bei einer 40jährigen Frau.

Was die Genese der in unserem Fall gefundenen Mißbildung anbelangt, so verweise ich auf die Ausführungen von $\mathrm{Ker-}$ m a u n e r. Die Genese des Prolapsus vesicae unseres Falles ist ohne weiteres klar: Die wiederholte und dabei lange Zeit erhöhte Bauchpresse infolge der Pertussis hat den Blasenvorfall bei dem mit Epispadie und Spaltbecken behafteten Mädchen veranlaßt.

\section{Literaturverzeichnis,}

I. B un ke, Zur Lehre von Inversion und Prolapsus der Blase. Jahrbuch für Kinderheilkunde 1889 , S. 418.

2. Crosse, Transact, of the Provincial Associat. I846. Vol. XI. Zit. nach Gurlt. Archiv f. klin. Chirurgie I864, Bd. 5, S. 366.

3. D u rand, L'épispadias chez la femme. Annales de Gyn. 1895, II. Zit. nach Rasch. Zur Kenntnis und Behandlung der weiblichen Epispadic. Beiträge f. klin. Chirurgie I 897 , Bd, 28 , S. 560 .

4. Fr u a, Ein Fall von Prolaps der Harnblase bei einem Säuglinge. The Lond. med. recod. 125. Annali univers, di med. 1876. Ref. Jahrbuch für Kinderheilkunde 1876 , S. 2 I 3 .

5. I m e rvol, De la hernie de la premiere enfance. Archiv de medec. des enfants I909, Bd. I2, S. I I5. Ref. Jahrbuch für Kinderheilkunde Igog, S. 622. 
6. Kerma u n r, Dic Mißbildungen des Rumpfes. Schwalbes Morphologie der Mißbildungen des Menschen und der Tiere. Teil III, Lieferung I, Igog, S. $4 \mathrm{I}$.

7. L owe, Lancet. I 862. Vol. I, S. 250 . Zit. nach Gurlt. 1. c. S. 365 .

8. Oliver, Inversion of Bladder. Edinb. med. Journ. Feb. S. 769. Ref. Jahresbericht der gesamten Medizin, Jahrgang X, Bd. 2, 1875, S. $25 \mathrm{I}$.

9. Rasch, Zur Kenntnis und Behandlung der weiblichen Epispadie und Fissura vesicae inferior. Beiträge zur klin. Chirurgie 1897, Bd. 18, S. 557.

10. Th o m s on, Cace of inversion of the bladder in an adult over forty years of age. Lancet Jan. 9. Ref. Jahresbericht der gesamten Medizin. Jahrgang $\mathrm{X}, 1876, \mathrm{Bd} .2$, S. $25 \mathrm{r}$.

I1. Weinlechner, Zur Lehre der Inversion des Prolapsus und der Invagination von Eingeweiden, Jahrbuch für Kinderheilkunde 187, S. 52 . 